\title{
A novel mutation in KRT12 associated with Meesmann's epithelial corneal dystrophy
}

\author{
A D Irvine, C M Coleman, J E Moore, O Swensson, S J Morgan, J H McCarthy, \\ F J D Smith, G C M Black, W H I McLean
}

Br J Ophthalmol 2002;86:729-732

Background: The molecular basis of Meesmann's epithelial corneal dystrophy (MECD) has recently been attributed to mutations in the cornea specific keratin genes KRT3 and KRT12. The mechanisms by which these mutations cause the Meesmann's phenotype are not clear. This study presents new data, examines clinical, histological, ultrastructural, and molecular aspects of MECD, and compares the features seen in this condition with those observed in other well studied keratin diseases such as epidermolysis bullosa simplex.

Methods: A two generation family with typical features of Meesmann's epithelial corneal dystrophy (MECD) was studied. All family members were examined under a slit lamp. Biopsy material from elective keratoplasty was studied by histopathological and ultrastructural analysis using standard techniques. Direct automated sequencing of genomic DNA was used for mutation detection, mutations were confirmed by restriction digest analysis.

Results: The abnormal corneal epithelium was acanthotic and contained numerous dyskeratotic cells and intraepithelial vesicles. By electron microscopy abnormally aggregated and clumped keratin filament bundles were detected in basal and suprabasal keratinocytes from the centre of the cornea. Direct sequencing of the patients' genomic DNA revealed a novel missense mutation (423T>G) in exon 1 of the cornea specific keratin 12 (KRT12) gene. This mutation predicts the amino acid change N133K within the helix initiation motif of the K12 polypeptide. Comparative studies with well established keratin disorders of other human epithelia underscore the pathogenic relevance of K3 and K12 gene mutations in Meesmann's epithelial corneal dystrophy. The morphological data presented here illustrate the disruptive effects of keratin gene mutations on the integrity of corneal keratinocytes.

Conclusions: A clinical, histopathological, and ultrastructural study of a previously unreported family with MECD is presented. In this family the disease is ascribed to a novel mutation in KRT12. A molecular mechanism is proposed for MECD based on the comparison with other well characterised keratin diseases.

M eesmann's epithelial corneal dystrophy (OMIM 122100; MECD $)^{1}$ is inherited as an autosomal dominant trait and was initially described in a German family. ${ }^{2}$ MECD is a bilaterally symmetrical disorder of the corneal epithelium with a characteristic slit lamp appearance of myriad fine round epithelial cysts of uniform size and shape, which become visible by 12 months of age and increase in number throughout life. ${ }^{3-5}$ Patients are usually asymptomatic until adulthood when rupture of the corneal microcysts may cause erosions, producing clinical symptoms such as photophobia, contact lens intolerance, intermittent diminution of visual acuity and, in some cases, permanent visual impairment.

Keratins are expressed specifically in the cytoplasm of epithelial cells where they form a dense meshwork of $10 \mathrm{~nm}$ intermediate filaments. ${ }^{6}$ Keratins are expressed as obligate heterodimers of type I/type II pairs in a tissue and differentiation specific fashion..$^{7-10}$ In 1991, the skin blistering disorder, epidermolysis bullosa simplex (EBS) was recognised as a human keratin disease. In EBS, mutations in either K5 or K14 genes render basal epidermal keratinocytes less resilient to mechanical stress, leading to increased skin fragility, which is manifest as blisters. ${ }^{11-13}$ Following this initial breakthrough, the total number of keratin genes associated with diseases (of the skin, hair, oral mucosa, and cornea) has risen to $18 .{ }^{10}$ The highly specialised corneal epithelium has evolved to combine the key function of providing structural protection to the eye while maintaining transparency. In keeping with this requirement, the keratin filament network of corneal keratinocytes appears less tightly bundled than that of cornified stratified epithelium such as the epidermis. ${ }^{14} \mathrm{~K} 5$ and K14 are expressed in limbal cells, ${ }^{14}$ but $\mathrm{K} 5$ and K14 mRNAs are undetectable beyond the limbus. ${ }^{15}$ As corneal keratinocytes approach terminal differentiation, $\mathrm{K} 3$ and $\mathrm{K} 12$, first expressed in the three to four layered developing cornea at 12-13 weeks' gestation, ${ }^{16}$ become the dominant keratin pair in all layers of the mature corneal epithelium. ${ }^{8}{ }^{15} 17$

The molecular basis of MECD was elucidated when the first mutations in the cornea specific keratins $\mathrm{K} 3$ and K12 were reported in three families, including Meesmann's original family. ${ }^{18}$ Subsequently, another group has confirmed these findings with mutations in K12 in four families with MECD. ${ }^{19}$ Here we report a clinical, histological, ultrastructural, and molecular study of a previously unreported family with MECD.

\section{MATERIALS AND METHODS}

A two generation family with three affected individuals was studied. All individuals were examined under a slit lamp by an experienced ophthalmologist (SJM/GCMB). Corneal tissue was obtained from the proband following lamellar keratoplasty. Samples were processed for routine histology and transmission electron microscopy. All subjects were treated in accordance with the tenets of the Declaration of Helsinki, and local institutional ethics committee approval was obtained. Specimens obtained at keratoplasty were fixed in formalin, placed in 10\% formal saline and processed on a Shandon Hypercentre. Paraffin wax embedded sections were cut at 3-4 $\mu \mathrm{m}$ and cut sections stained with haematoxylin and eosin, periodic acid Schiff, and Masson's trichrome. For electron microscopy, specimens were fixed in $2 \%$ glutaraldehyde in Sörensen's buffer and then $2 \%$ osmium tetroxide using a standard protocol. Semithin $(1 \mu \mathrm{m})$ sections were cut on an Ultracut E (Reichert-Jung) and stained with toluidine blue. 


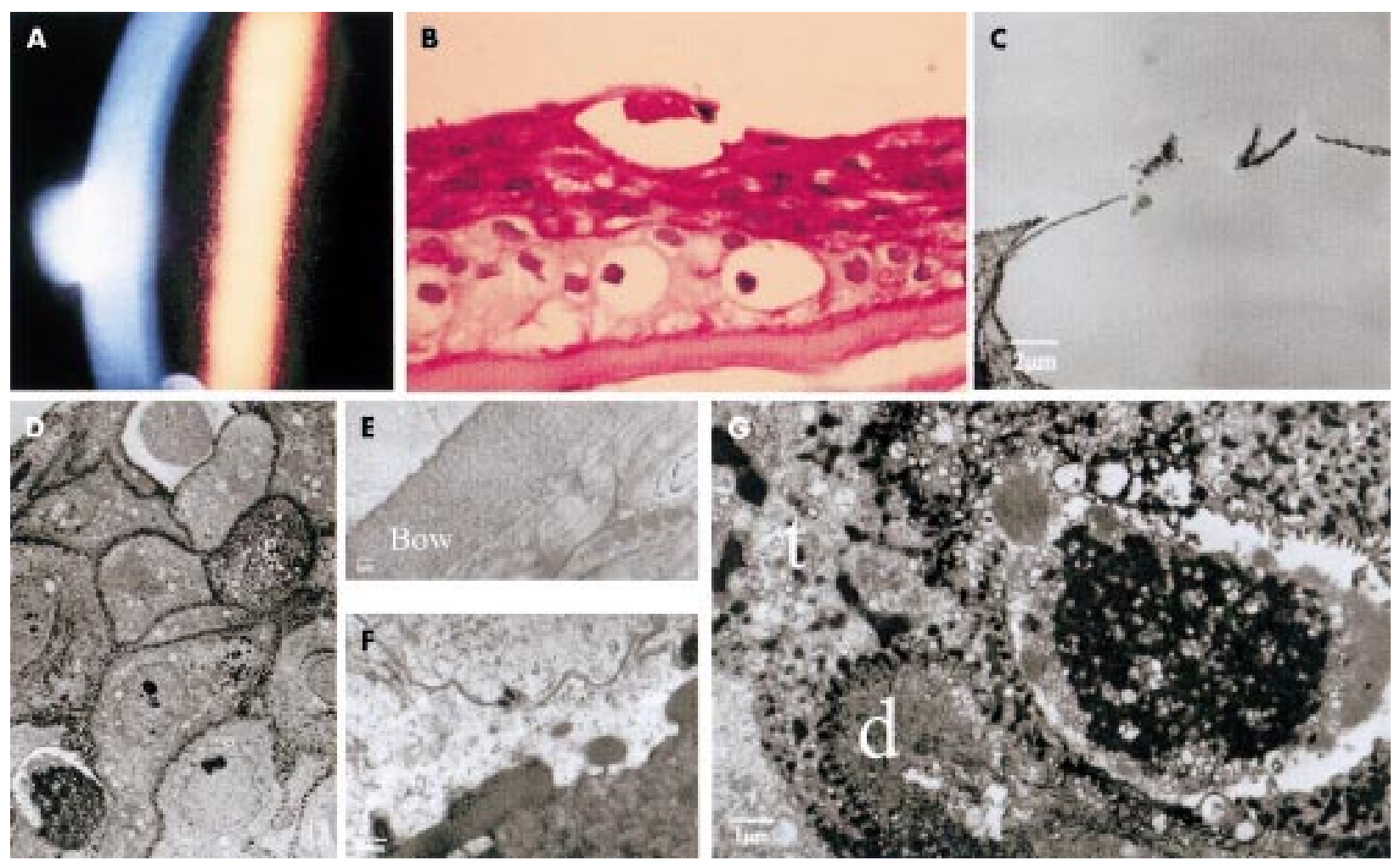

Figure 1 (A) Characteristic pretreatment of multiple small cysts seen on slit lamp examination. (B). Light micrograph showing abnormal corneal epithelium of the proband. Bowman's membrane presents as a homogeneous eosinophilic subepithelial band. The epithelium appears acanthotic and disordered. Many keratinocytes contain periodic acid Schiff (PAS) positive fibrillar material (PAS stain, $\times 200)$. (C-F) Transmission electron micrographs illustrating formation of intraepithelial vesicles in MECD. (C) Intraepithelial vesicle. Thinned and discontinuous cell membranes incompletely separate the vesicle lumen from the corneal surface, bar $=2 \mu \mathrm{m}$. (D) Keratinocytes of the outer epithelial layers vary remarkably in size, shape and electron density. A single dyskeratotic cell is seen. Note the lack of acantholysis indicative of largely intact intercellular contacts. (E) and (F) Basement membrane ultrastructure. (E) The basement membrane has a thickened appearance, bar $=1 \mu \mathrm{m}$. (F) A higher power view confirms basement membrane thickening and shows the striking feature appearance of breaches or gaps within the basement membrane, bar $=500 \mathrm{~nm}$. (G) Detail of dyskeratotic cell. Note marked perinuclear vacuolisation and cytoplasmic condensation. The keratin intermediate filaments in MECD are shortened, thickened, and clumped and appear to be associating with desmosomes, bar $=1 \mu \mathrm{m}$. Bow, Bowman's membrane; $t$, tonofilament; $d$, desmosomes.

Tissue was embedded in Araldite resin (Araldite resin embedding kit-E009 Taab). Electron microscopy was performed on a Philips EM410.

Most of exon 1 of the KRT12 gene was amplified from affected and unaffected family members using primers K12el.L (5' ATG GAT CTC TCC AAC AAC ACC ATG 3') and K12el.R (5' GTA ATC GCT CTG TGA AGC ATC TGC 3'), as described previously. ${ }^{18}$ Polymerase chain reaction (PCR) products were purified using QIAquick columns (Qiagen, Chatsworth CA, USA), sequenced with both primers using the ABI PRISM Ready Reaction system (Perkin-Elmer, Foster City, CA, USA) and analysed on an ABI 377 automated sequencer. The 423T $>\mathrm{G}$ mutation generates an Afl II restriction site. To confirm the mutation within the family and exclude it from 100 normal unrelated chromosomes, PCR products generated with primers K12spl (5' CTC TAG GTA TTC TCT CGG GCA ATG $\left.3^{\prime}\right)$ and K12sp2 (5' TGC TGC AAG TAC AGC TAA ATT GGA 3') were digested overnight with $1.5 \mathrm{U}$ Afl II and analysed on 3\% Seakem/TBE minigels.

\section{RESULTS AND DISCUSSION}

All affected individuals had the characteristic slit lamp appearance of multiple fine round epithelial cysts (Fig lA). Individuals in the second generation had a relatively symptom free presentation. The proband had a more problematic clinical course with a history of soreness in both eyes from his late teens, especially marked in the morning. The symptoms had progressed with age, necessitating medical intervention. At age 40 he noted gradual bilateral deterioration in visual acuity and bilateral epithelial debridement was performed at age 41 years. Unfortunately he experienced recurrence of symptoms and clinical signs within 1 month. Excimer laser PTK was performed to the left (amblyopic) eye, but again there was recurrence of symptoms within 1 month. Because of persisting symptoms a non-matched lamellar keratoplasty to left eye was performed with relief of symptoms for 3 months, but full recurrence within 3 months. At age 45 years he underwent donor limbal stem cell allograft to the left eye with fresh, nonmatched, whole eye as source. This was initially successful, with only minor clinical recurrence despite clinical evidence of rejection. Subsequently, he underwent donor limbal stem cell allograft to the right eye from a tissue matched donor. This eye pursued a similar course to the left. Eventually, his symptoms recurred necessitating long term bandage therapeutic contact lens wear, although his corneas still only showed a moderate degree of clinical recurrence.

By light microscopy abnormal epithelium from the centre of the cornea was thickened and comprised five to six cell layers (Fig 1B). The overall architecture of the epithelium seemed disordered with many keratinocytes varying in size and shape. A number of cells showed marked vacuolisation and dense intracytoplasmic inclusions. In addition, numerous intraepithelial vesicles were noted in the outer layers of the epithelium. Ultrastructural changes were noted in all layers of the corneal epithelium and most prominent changes were seen in the outer epithelial layers, with thin walled vesicles clearly apparent (Fig 1C). The cytoplasm of many keratinocytes varied in size, shape, and electron density (Fig 1D). Overt 

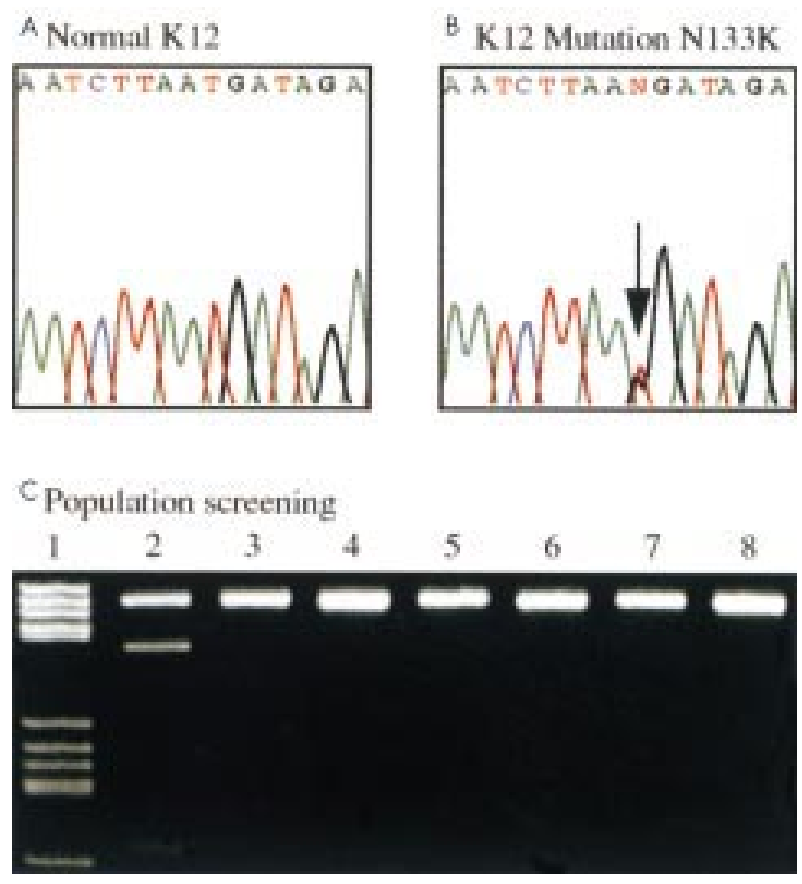

Figure 2 Molecular genetic analysis. (A) and (B) KRT12 mutation detection by direct automated sequencing of genomic PCR products (A) Normal KRT12 exon 1 sequence, showing codons 131-135. (B) The equivalent sequence shown in $(A)$ derived from the proband. $A$ heterozygous transversion mutation $423 T>G$ is shown (arrow). This mutation predicts the amino acid substitution N133K in helix $1 \mathrm{~A}$ of the K12 polypeptide. (C) Mutation N133K creates a new Afl II restriction site. Therefore, the mutation was confirmed in the proband and excluded from normal controls by Afl II digestion of KRT12 exon 1 PCR products. Lane 1, molecular weight markers; lane 2, digest derived from the proband showing both uncut and cut fragments corresponding to the normal and mutant alleles, respectively; lane 3-8, digests of KRT12 exon 1 PCR fragments derived from six normal, unrelated individuals. By this means, the mutation was excluded from a total of 50 normal people (data not shown).

acantholysis was not seen. The outer layers of the epithelium contained numerous dyskeratotic cells with condensed nuclei, perinuclear vacuoles, and rim-like profiles of condensed cytoplasm. Dyskeratotic cells in the outermost epithelial layers seemed to precede the formation of intraepithelial vesicles. The basement membrane was thickened (Fig 1E), confirming the impression gained from light microscopy. And several breaches were noted at higher power (Fig IF). Numerous cells showed intracytoplasmic vacuoles and abnormal, aggregated keratin filaments. The keratin clumps tended to form oval and round intracytoplasmic inclusions, some of these aggregates appeared to be associated with desmosomes (Fig lG).

A novel heterozygous $\mathrm{T}>\mathrm{G}$ transversion mutation at nucleotide position 423 in exon 1 of the KRT12 gene (designated $423 \mathrm{~T}>\mathrm{G}$ ) was demonstrated by direct sequencing (Fig 2A). The mutation predicts the amino acid substitution N133K in the K12-polypeptide. This change affects residue eight of helix $1 \mathrm{~A}$ and would be classified as N8K in relation to this domain. The $423 \mathrm{~T} \rightarrow \mathrm{G}$ transversion generates an Afl II restriction site, allowing confirmation by restriction digest analysis (Fig 2B). By this means, the mutation was excluded from a population of 50 normal unrelated individuals. Thus, the mutation is unlikely to represent a common polymorphism.

Meesmann's epithelial corneal dystrophy (OMIM No 122 100; MECD) ${ }^{1}$ was initially described by Pameijer in 1935 in an 8 year old Dutch boy. ${ }^{2}$ Later, Meesmann studied three large German families and reported the clinical phenotype and characteristic histopathological features. ${ }^{3}$ Following these initial reports MECD has been documented in many other populations. The inheritance pattern of MECD has consistently been autosomal dominant. ${ }^{2-5}$ 18-23 $^{-3}$ Multiple epithelial microcysts become visible in the first few months of life under slit lamp magnification, ${ }^{24}$ but MECD often remains asymptomatic throughout life. However, in a number of individuals, it first manifests itself during adolescence with recurrent punctate erosions, excess lacrimation, photophobia, and blepharospasm. Visual acuity may be temporarily diminished during erosive episodes or permanently diminished if there is resultant corneal scarring. Slit lamp appearances of myriad fine round cysts are seen most prominently in the interpalpebral zone (Fig lA), serpiginous grey lines and small subepithelial opacities may be found in advanced stages. ${ }^{18}$ While the index case in this report suffered from the most severe manifestations of MECD, it remains to be seen if his children, who are currently in their teenage years will develop a condition of similar severity. Intrafamilial variation between individuals is well described in MECD and in other keratin diseases, ${ }^{10}$ which suggests the influence of disease modifying genes and/or environmental influences. Corneal debridement often leads to clinical improvement; however, the corneal stem cells retain the mutated genes and so the cysts and symptoms can recur.

While having some unique features, which presumably reflect the highly specialised nature of the corneal epithelium, MECD has many features in common with other well characterised keratin diseases affecting other epithelia. The multiple cysts seen clinically on slit lamp examination are analogous to the intraepithelial cytolysis in other keratin disorders but are seen more easily in the transparent corneal epithelium. The histological appearance of thickened and disorganised epithelium seen in MECD is characteristic of a keratin disorder. ${ }^{10}$ The cytolysis and cell degeneration seen histologically in all keratin disorders is manifest in the cutaneous context as epidermolysis (in EBS, bullous congenital ichthyosiform erythroderma and ichthyosis bullosa of Siemens). Here, in the corneal context, cytolysis is manifest as cyst formation. We believe the PAS positive clumps noted by previous authors and demonstrated in this study represent aggregates of keratin filament clumps and associated cellular debris. Although keratins are not generally regarded as glycoproteins, it has been shown that keratins $\mathrm{K} 8$ and K18 have short O linked sugar side chains. ${ }^{25}$ Glycosylation of $\mathrm{K} 3$ and $\mathrm{K} 12$ has not been reported. In addition, intermediate filament aggregates have been shown to contain chaperone proteins of the small heat shock family, ${ }^{26}{ }^{27}$ offering another explanation for the PAS reactivity observed. Unfortunately, frozen biopsy material was not available to confirm this association by immunohistochemistry.

The ultrastructural observation of tonofilament clumping in MECD is again reminiscent of findings in other keratin diseases. In the most comprehensively studied human keratin disease, the Dowling-Meara form of EBS (EBS-DM), ultrastructural tonofilament clumping was first suggested as being indicative of keratin disease as early as $1982,{ }^{28}$ and these clumps were formally identified as being composed of K5/K14 aggregates by immunohistochemistry in 1991. ${ }^{29}$ The clumped tonofilaments seen here are presumably $\mathrm{K} 3 / \mathrm{K} 12$ aggregates although we were unable to confirm this by immunoelectron microscopy owing to the method of preparation of the archival tissue. The multiple dyskeratotic cells are not seen in other keratin disorders and presumably reflect the unique nature of the corneal epithelium. The appearance of breaches in the basement membrane is also atypical.

In conclusion, MECD shares many molecular, histological, and ultrastructural features with other keratin disorders but also has specific characteristics, probably because of the unique morphology of the anterior corneal epithelium. Further work to more clearly define the mechanisms underlying the development of the "peculiar substance" is planned. This will shed light on the role of cornea specific keratins in the maintenance of epithelial integrity and in the aetiology of conditions associated with its breakdown. 


\section{Authors' affiliations}

A D Irvine, C M Coleman, F J D Smith, W H I McLean, Human Genetics Unit, University of Dundee, Dundee, UK

J E Moore, Department of Ophthalmology, Royal Group of Hospitals, Belfast, UK

O Swensson, Department of Dermatology,

Christian-Albrechts-Universität, Kiel, Germany

S J Morgan, Sunderland Eye Infirmary, Sunderland, UK

J H McCarthy, South Tyneside Healthcare Trust, Harton Lane, South Shields, UK

G C M Black, Manchester Royal Eye Hospital/Department of Clinical Genetics, Manchester, UK

Correspondence to: Alan D Irvine, MD MRCP, Human Genetics Unit, University of Dundee, Ninewells Hospital and Medical School, Dundee DD1 9SY, UK; irvo67@hotmail.com

Accepted for publication 18 February 2002

\section{REFERENCES}

1 McKusick VA. OMIM (On-line Mendelian Inheritance in Man). 2002 release ed, 2002

2 Pameijer JK. Über eine fremdartige familiare oberflächliche Hornhautveränderung. Klin Monatsbl Augenheilkd 1935;103:516-17.

3 Meesmann A, Wilke F. Klinische und anatomische Untersuchungen veber eine bisher unbekannte, dominant vererbte Epitheldystrophie der Hornhaut. Klin Monatsbl Augenheilkd 1939;103:361-91.

4 Burns R, P. Meesmann corneal dystrophy. Trans Am Ophthalmol Soc 1968;66:630-5.

5 Fine BS, Yanoff M, Pitts E, et al. Meesmann's epithelial dystrophy of the cornea. Am J Ophthalmol 1977;83:633-42.

6 Quinlan RA, Hutchison CJ, Lane EB. Intermediate filaments. In: Sheterline P, ed. Protein profiles. London: Academic Press, 1994.

7 Fuchs E, Green H. Changes in keratin gene expression during terminal differentiation of the keratinocyte. Cell 1980;19:1033-42.

8 Sun T-T, Eichner R, Schermer A, et al. Classification, expression and possible mechanisms of evolution of mammalian epithelial keratins: a unifying model. New York: Cold Spring Harbor Laboratory, 1984.

9 Lane EB. Keratins. In: Royce PM, Steinmann B, eds. Connective tissue and its heritable disorders. Molecular, genetic and medical aspects. New York: Wiley-Liss, 1993:237-47.

10 Irvine AD, McLean WHI. Human keratin diseases: increasing spectrum of disease and specificity of genotype-phenotype correlation. $\mathrm{Br} J$ Dermatol 1999;140:815-28.

11 Bonifas JM, Rothman AL, Epstein EH. Epidermolysis bullosa simplex: evidence in two families for keratin gene abnormalities. Science 1991;254:1202-5.
12 Coulombe PA, Hutton ME, Letai A, et al. Point mutations in human keratin 14 genes of epidermolysis bullosa simplex patients: Genetic and functional analysis. Cell 1991;66:1301-11.

13 Lane EB, Rugg EL, Navsaria H, et al. A mutation in the conserved helix termination peptide of keratin 5 in hereditary skin blistering. Nature 1992;356:244-6.

14 Kao WW-Y, Liu C-Y, Converse RL, et al. Keratin 12 deficient mice have fragile corneal epithelia. Invest Ophthalmol Vis Sci 1996;37:2572-84.

15 Wu RL, Zhu G, Galvin S, et al. Lineage-specific and differentiation-dependent expression of K12 keratin in rabbit corneal/limbal epithelial cells: cDNA cloning and northern blot analysis. Differentiation 1994;55:137-44.

16 Rodrigues M, Ben-Zvi A, Krachmer A, et al. Suprabasal expression of a 65-kDa keratin (No.3) in developing human corneal epithelium. Differentiation 1987;34:60-7.

17 Schermer A, Galvin S, Sun T-T. Differentiation-related expression of a major $64 \mathrm{~K}$ corneal keratin in vivo and in culture suggests limbal location of corneal epithelial stem cells. J Cell Biol 1986;103:49-62.

18 Irvine AD, Corden LD, Swensson O, et al. Mutations in cornea-specific keratins K3 or K1 2 cause Meesmann's corneal dystrophy. Nat Genet 1997:16:184-7.

19 Nishida K, Honma Y, Dota A, et al. Isolation and chromosomal localization of a cornea-specific human keratin 12 gene and detection of four mutations in Meesmann corneal epithelial dystrophy. Am J Hum Genet 1997;61:1268-75.

20 Kuwabara T, Ciccarelli EC. Meesmann's corneal dystrophy: a pathological study. Arch Ophthalmol 1964;71:676-82.

21 Theil H-J, Behnke H. Über die Variationsbreite der hereditären Hornhautepitheldystrophie (Typ Meesmann-Wilke). Ophthalmologica 1968;155:81-6.

22 Pülhorn G, Theil H-J. Licht- und elektromenmikroscopische Untersuchungen über die Zystenbildung bei hereditärer Hornhautepitheldystrophie Meesmann-Wilke. Ophthalmologica 1974; 168:348-59.

23 Tremblay M, Dube I. Meesmann's corneal dystrophy: ultrastructural features. Canad J Opthalmol 1982;17:24-28.

24 Snyder WB. Hereditary epithelial corneal dystrophy. Am J Ophthalmol 1963;55:56-63.

25 Ku NO Omary MB. Expression, glycosytlation, and phosphorylation of human keratins 8 and 18 in insect cells. Exp Cell Res 1994;211:24-35.

26 Perng MD, Cairns L, van den IP, et al. Intermediate filament interactions can be altered by HSP27 and alphaB-crystallin. J Cell Sci 1999; 112:2099-112

27 Van den lissel P, Norman DG, Quinlan RA. Molecular chaperones: small heat shock proteins in the limelight. Curr Biol 1999;9:R103-5.

28 Anton-Lamprecht I, Schnyder UW. Epidermolysis bullosa herpetiformis Dowling-Meara: report of a case and pathomorphogenesis. Dermatology 1982;164:221-235.

29 Ishida-Yamamoto A, McGrath JA, Chapman SJ, et al. Epidermolysis bullosa simplex (Dowling-Meara type) is a genetic disease characterized by an abnormal keratin filament network involving keratins $\mathrm{K} 5$ and $\mathrm{K} 14$. J Invest Dermatol 1991;97:959-68. 